\title{
SPIGELIAN HERNIA REPAIR USING THE PROLENE HERNIA SYSTEM
}

\author{
By \\ EISSA ABDEL HAMED IBRAHIM ${ }^{1}$ and YASSR MOHAMED SHAFIK ${ }^{2}$ \\ Consultant General Surgery ${ }^{1}$, and Consultant Anesthesia and Pain Management ${ }^{2}$ \\ Military Medical Academy, Cairo 11291, Egypt \\ ( ${ }^{*}$ Correspondence:eissaabdelhameed@gmail.com) \\ Abstract
}

The results of spigelian hernia repair with prolene hernia system in a prospective study were evaluated from October 2014 to December 2018 on 20 patients with spigelian hernia- 12 patients female and 8 patients male were operated using prolene hernia system (prolene mesh) repair. All had spigelian hernia most of the hernia located on right side. All patients had examination 2 week after operation and follow up every month up to one year, without major complication. There was three miled wound infection and was treated by antibiotic and that improved without removing the mesh. No recurrence after the operation sermoa was found in four patients three female and on male and was treated by aseptic aspiration of seroma and antibiotic. Mean hospital stay was 2 days. Because of low complication rate, no recurrent and short hospital stay spigelian hernia repair with the prolene hernia system is the operation of choice for spigelian hernia.

Keywords: Egypt, Spigelian Hernia, Prolene Hernia System.

\section{Introduction}

Spigelian Hernia: Spigelian fascia is the aponeurotic part of the transverses abdominal muscle between the medial border of its muscular part and the insertion of the aponeurosis into the posterior rectus sheath by definition, it's a variety of interparietal hernia occurring at the level of arcuate line (Baily and Love's, 2000).

Mean age was 50 years, ratio of females to males was $1.4: 1$, ratio of hernia on right side to that on left side was $1.6: 1$, bilateral hernia was $0.75 \%$, most of them located below the umbilicus level, incarceration at the operation time occurred in $21.2 \%$, in $1 \%$ hernia sac situated subcutaneously in most cases hernia was located between musculo-poneurotic layers of anterior abdominal wall.
Anatomy: Semilunar line that marks the lateral border of the rectus sheet, rectus sheath stretches from the tip of the ninth rib cartilage to the pubic tubercle, the spigelian fascia, which is really a strip of apponeurosis runs parallel and lateral to the outer border of the rectus sheath. Its fibers fuse with those of the internal and external oblique muscle apponeurosis to from the rectus sheath. Spigelian fascia: the spigelian fasica varies in width; a- At its upper end its hardly exists since the muscular part of transverses abdomens usually reaches up to the semilunar line, and b- It gets wider as it passes down to the umbilicus level and is widest in that part between the level of umbilicus and meets the arcuate fold of Douglas (Fig. 1).



Fig. 1: Spigelian hernia. A: Sites of spigelian hernia and sections through normal body wall above and below umbilicus, showed semilunar "zone" of spieghel. B: Relation of a spigelian hernia to inguinal structures, a, artery (Skandallkis et al, 1974). 
Read (1979) described the insertion of the transverses abdominal and internal oblique muscles into the rectus sheath as series of microbundle of muscle and microtendons in a parallel arrangement with gaps between them that are filled by fibro-fatty septae. The usual protective gridiron arrangement of the abdominal wall musculature did not exist here so that factors such as: aging of the tissues, obesity, increased abdominal pressure due to (coughing, constipation, prostatism, sudden lifting of a heavy weight, pregnancies). It may force the per-peritoneal fat via the fibro-fatty septae and thus caused a spigelian hernia.

Most of the hernias occur in the area between the level of the umbilicus and the level of the arcuate line or fold of douglas where the spigelian fascia is widest and presumably weakest. The hernias occur particularly at the point where the semilunar and arcuate line meet since it is at this point that all the fibers of the transversus abdominal muscle pass in front of the rectus muscle. There is no posterior rectus sheath below this point. The rearrangement of the fibers at this point is believed to cause an area of functional weakness where the hernias can more easily occur (Fig. 2). Hernias at the upper reaches of the semilunar line are not usually true spigelian hernias since there is rarely any spigelian fascia there. These hernias usually pass through the posterior rectus sheath along the medial side of the semilunar line and expand in the rectus sheath and are called intravaginal hernias.

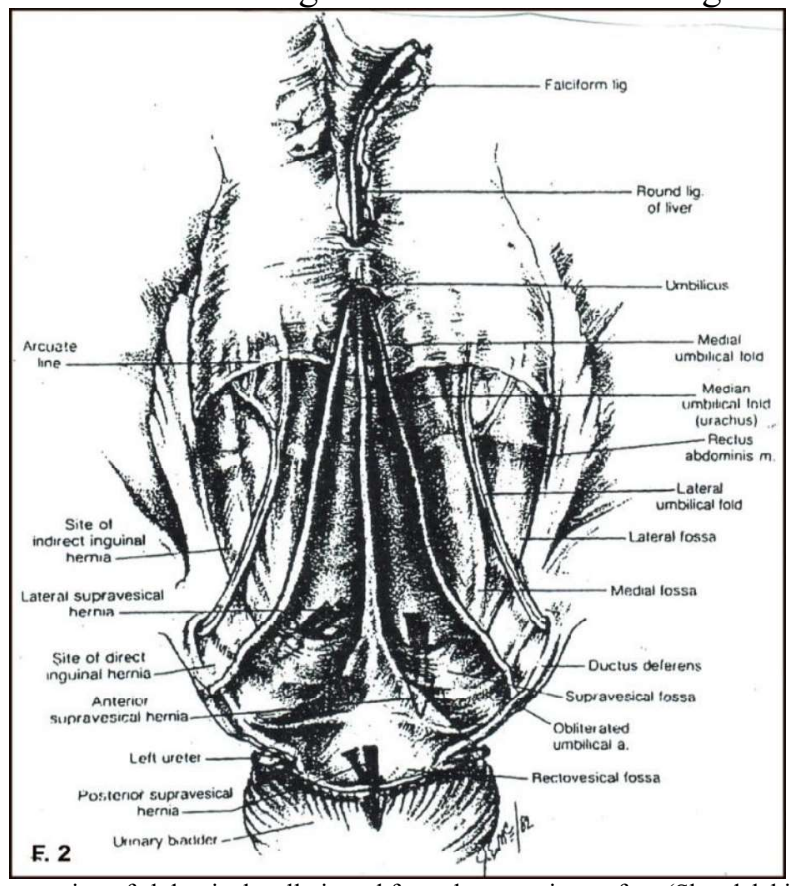

Fig. 2: Anterior portion of abdominal wall viewed from the posterior surface (Skandelakis et al, 1974).

Hernias below the level of the arcuate line of douglas pass through the conjoined tendone of the trasnversus abdominis and intenal oblique muscles and are called "low" spigelian hernias at the lowest levels they may behave like direct inguinal hernias.

As the hernia develops pre-peritoneal fat pushes its way through the slit-like defects of the spigelian fascia and the aponeurosis of the internal oblique muscle dragging a protrusion of peritoneum with it "it is virtually impalpable". Hernia meets resistance from aponeurosis of external oblique muscle but is able to mushroom in loose areolar tissue between internal oblique and external oblique muscle and gives rise to a more evident swelling. Due to anatomic variations a small number of hernias pass via aponerusis 
of transversus abdominal muscle and lie between that muscle and the internal oblique muscle. Almost all spigelain henias are interstitial (interparietal). Rarely, hernia may pierce the aponeurosis of the external oblique muscle and lie in subcutaneous tissues of the abdominal wall. It is unable to spread medially because of the rectus sheath resistance but it can develop laterally and inferiorly and lie alongside the anterior superior iliac spine or even in inguinal region (Fig. $3)$.



Fig. 3: Three-dimension schematic presentation of cross-section of abdominal wall in vicinity of left border of the sheath of rectus abdominus muscle, posterior view. A: Above semicircular line of douglas, B: Below semicircular line of douglas. Spigelian hernia sac is shown in various surgical levels: I- Superfacial to aponerurosis of transverse abdominal muscle, II- Superfacial to aponeurosis of internal oblique, IIISuperficial to openurosis of external oblique muscle, IV- Penetrating posterior lamina of rectus sheath (Skandalakis et al, 2006).

Clinical manifestations: Patients complain of: a- Pain, b- Lump at site of herniation, or c- both. The pain is sharp and constant or intermittent or there is a dragging uncomfortable feeling. It may be aggravated by (Coughing, straining, standing, stretching the arms above the head, long hours of physical work). If strangulation of the hernia contents is present the pain will be severe and constant and associated with symptoms and signs of complete or partial (Richter) intestinal obstruction going on to gangrene and peritonitis. Localized perforation into the sac may cause an abdominal wall abscess and even fistula.

When a mass is present along the somilunar line especially in the region below the umbilicus the diagnosis becomes easier. The mass may be reducible after which the hernia defect in the fascia may felt. There is usually tenderness present in the defect region. Palpable mass of a spigelian hernia whether it be subcutaneous or interstitial usually persists during abdominal contraction.
When hernia is incarcerated and irreducible it may confuse with a- Lipoma, b- Despoid tumor, and c-Hematoma of the rectus sheath or even an appendix abscess (Fig. 4), in confirming diagnosis by showing bowel outside the abdominal cavity at the site pain tenderness or mass

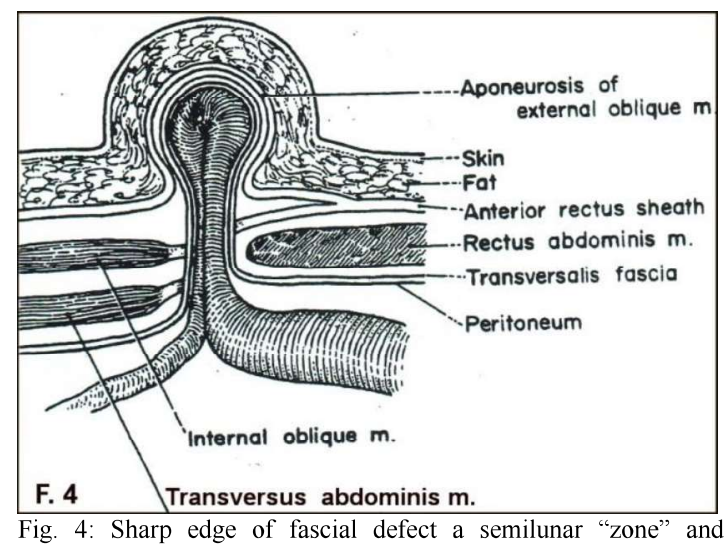
spigelian hernia sac penetrating it (Skandalakis et al, 1974).

Plain x-ray films or with contrast media especially tangential views may be helpful.

Ultrasound examination is the best easiest and most reliable test available for diagno- 
sing spigelian hernia, if it is reduced without palpable mass ultrasound scanning showed a break in the echogenic shadow of the smilunar line corresponding to the fascial defect.

Spigelian hernia may be an elusive diagnosis and may mimic other intra-abdominal conditions (Andersen, 1986). The advantage of real time ultrasonography (Fig 5) is the ability to perform examination in both spine and upright positions and while patient performs a valsalve maneuver (Co-mpos and Waldeut 1994). The increasing availability of the magnetic resonance imaging (MRI) may be of benefit in the preoperative evaluation of difficult cases (Compos and Walden, 1997).

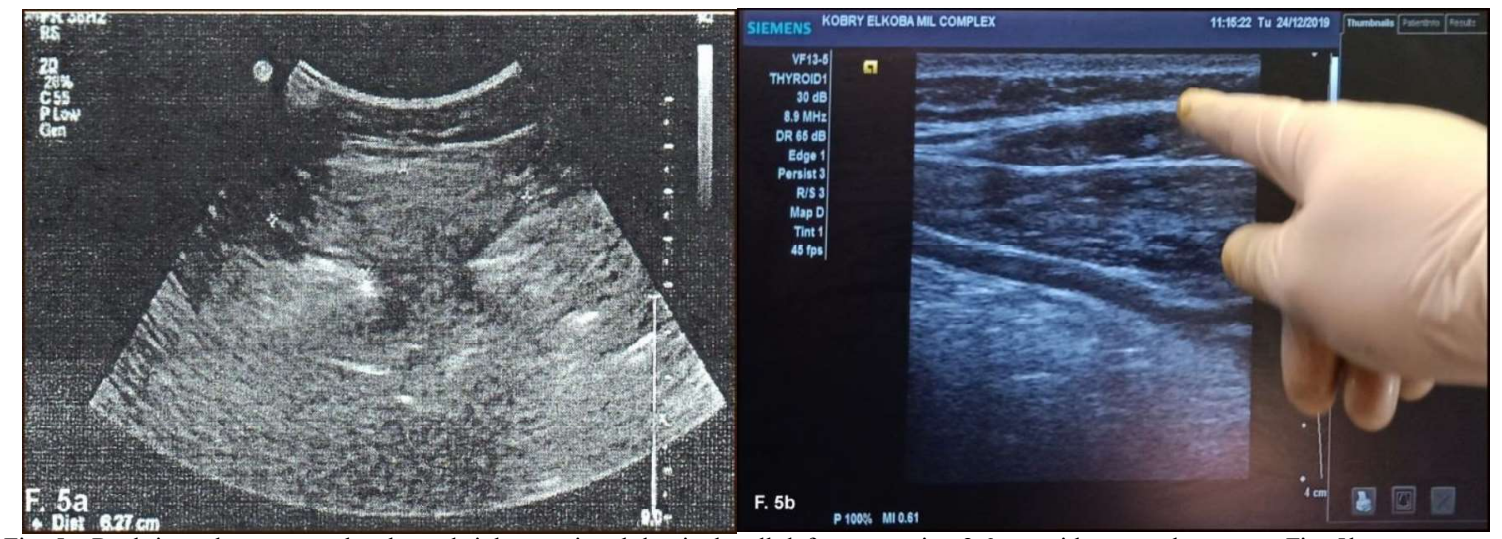

Fig. 5a: Real time ultrasonography showed right anterior abdominal wall defect averaging $2.6 \mathrm{~cm}$ with omental content Fig. $5 \mathrm{~b}$ Normal appearance of anterior abdominal wall and aspiglian zone below level of umbilicus (Kobbry El-Kobba Military Medical Campus, Radiologic department, 2018).

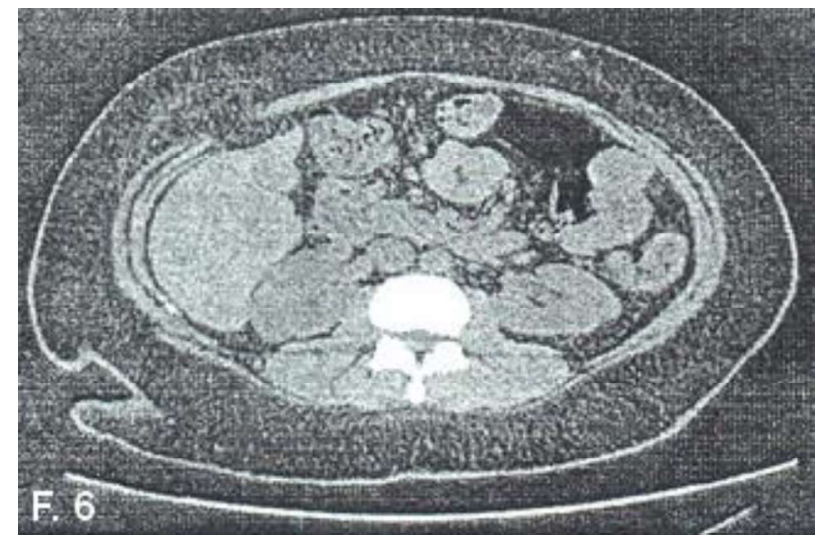

Fig. 6: CT showed defect of aspigelian hernia about $3 \mathrm{~cm}$ in diameter, containing omentum Kobbry El-Kobba Military Medical Campus, Radiologic department, 2018).

Treatment: At least $20 \%$ of spigelian hernias presented with strangulation. The proper treatment is operative repair which is more conveniently done with the patient under general anesthesia although local or regional methods "Transverses abdominal plane block (TAP)" could be used (Andersen, 1986).

Operative technique: A- Conventional approach: 1-A transverse or oblique skin incision is made over the lump or over the fascial defect. 2- A subcutaneous hernia will immediately reveal itself but more comm- only the hernia is interstitial and the external oblique muscle must be split in the line of its fibers to demonstrate the sac. 3- It is freed from surrounding tissues down to the neck. Sac was opened and its contents reduced back into peritoneal cavity. Some adhesions between the consents and the sac wall were freed, sac was excised or inverted without tie or suture it. 4- Defect in fascia of transverses abdominal and internal oblique muscle was closed with continuous suture of monofilament polypropylene. 5- Slit in external 
oblique muscle was similarly repaired, and 6- Subcutaneous fat and skin were approximated, the repair heals well. Few recurrences have been reported (Andersen, 1986).

B- Endoscopic approaches: Intraperitoneal on lay mesh repair: intra-peritoneal access was done using versus needle or open technique once abdominal access is obtained site of hernia orifice was readily identified and ports are placed at least $10 \mathrm{~cm}$ away from hernia defect in the form of an arc of a circle whose center in hernias defect.

Contents were reduced from sac and adhesolysis is performed if required to obtain on overlap of $\mathrm{cm}$ around defect for synthetic mesh, which was fixed using a combination of trans-abdominal sutures and tack (Mittal et al, 2008).

A. Trans-abdominal pre-peritoneal repair: When hernia sac contents were reduced a peritoneal flap raised as in trans-abdominal pre-peritoneal approach and attempt to completely reduce the hernia sac. After dissecting peritoneal flap, about $5 \mathrm{~cm}$ around hernia defect prolene mesh was placed in the dissected extra-peritoneal space and fixed by tacks. Peritoneal flap was closed either with tack or with a continuous suture (Carter and Mizes, 1992).

B. Total extra peritoneal repair: Endoscopic T.E.P repair is performed using 3 mid-line port extra-peritoneal space is created by open access and balloon. The spigelian hernia sec was identified around arcuate line and reduced completely. The peritoneum is dissected above the arcuate line to have a $5 \mathrm{~cm}$ margin around the hernia defect for mesh overlap. A prolene mesh covered the hernia defect. Mesh was fixed to anterior abdominal wall with spiral tacks (MorenoEgeo et al, 2002).

This study aimed to demonstrate surgical treatment using the prolene hernia system among spigelian hernia repair patients in Kobbry El-Kobba Military Medical Campus from October 2014 to December 2018.

\section{Patients and Methods}

The study was carried out on 20 patients with spigelian hernia suffered from chronic pain with or without lump. The patients were 12 patients female and 8 patients male, their age varied between 40 years old and 70 years old with mean age of 52 years old, 6 patients civeline and 14 patients military.

All patients suffered from spigelian hernia mainly at right side (16 patients) less common at left side (4 patients). Diagnosis of spigelian hernia was established by clinical examination and ultrasound up on muscle of anterior abdominal wall to detect defect (CT or MRI) on abdominal wall if need in dutiful patient, CT was done to 4 patients. All patients were subjected to full examinations of blood sugar liver enzymes profile, pre-thrombin time, INR, CBC, ECG, chest $\mathrm{x}$ ray and abdominal ultrasound.

Operative technique: 1- Marking the site of spigelian hernia by clinical examination, US and marking it by dot line on patient (Fig. 7). 2- Patients were anesthetized by general anesthesia and place in spine position. A transverse skin incision was made over the lump (Fig. 8) presented by dot line. A subcutaneous hernia immediately showed itself by sac demonstration (Fig. 8). 3- Sac was freed from surrounding tissues down to neck, opened and the contents reduced back into peritoneal cavity and excised sac and closed the defect in fascia transversals. Peritoneum was closed by continuous suture of monofilament polypropylene or vecryl (Fig. 9). 4- Create space between fascia transversalis and transverse abdominal muscle for insertion of circular part of prolene hernia system. After insertion of circular part of mash, defected fascia traverses were closed around connects part (connected part between-circular part and longitudinal part) by monofilament poly propylene (Figs. 10, 11, 12). 5Longitudinal part was sutured to external oblique openurosis (Fig. 13). 6- Wound closure by interrupted suture to subcuacnus fat 
and closure of skin using prolene subcuticular and insertion of radivac (Fig. 14).

All patients were given cephalosporin ${ }^{\circledR}$ $1 \mathrm{gm}$ IV in preoperative night $\& 1 \mathrm{gm}$ at anesthesia time; postoperative prophylactic anticoagulant (Clexan 40iu/24H). Radivac was removed 5 day's post operatively and skin suture 7 days in outpatients' clinic.

Operative time's post-operative hospital stay and postoperative complication were re- ported. Patients were routinely followed by physical examination in outpatient clinic 2 week after operation and follow up every month up to one year and then every year.

\section{Results}

Mean operative time for spigelian hernia repair was 35 (28->50) min-median postoperative hospital stay was 2 days $(1->5)$ days. There were no serious preoperative difficulties and no recurrence (Tab. 1).

Table 1: Post-operative complications.

\begin{tabular}{|l|l|}
\hline Complication & Number of patient \\
\hline - Postoperative death (0-15 days) & No \\
- Pulmonary complication & 1 \\
- Bleeding & 2 mal pation \\
- Seruma & No \\
- Wound infection (superfascial and deep wound infection) & 4 patients \\
\hline
\end{tabular}

One patient (65 years) showed mild chest infection and treated by antibiotic, two male patients showed postoperative urinary retention and treated by catheterization and $\alpha$ blocker due to serial enlarged prostate and seroma in 4 patients ( 3 females \& 1 male) and treated by aseptic aspiration of seroma and antibiotic, 4 patients showed mild superfacial wound infection and treated by antibiotic without wound opening nor mesh removal or deep wound infection.

\section{Discussion}

Spigelian hernia is a variety of inter-peritoneal hernia occurs in at the level of the arcuate line. It is very rare with only 1000 cases reported in the literature (Baily and Love's, 2000). Sac fundus was clothed by extra-peritoneal fat beneath internal oblique muscle where it was virtually impalpable.

More often it advances through that muscle and spreads out like a mushroom between internal and external oblique muscles and gives rise to a more evident swelling. The male patient was corpulent and over 50 years, but women were unequally affected.

Typically a soft, reducible mass was encountered lateral to rectus muscle and below umbilicus and diagnosis was confirmed by ultrasound scanning. The US has the ability to stand patient upright without visible defect in recompiling position. CT and MRI proved reliable in diagnosing difficult cases. Owing to rigid fascia surrounding the neck, strangulation were suspected.

\section{Recommendations}

1- Spigelian hernia may be an elusive diagnosis and manic other intra-abdominal condition US examination is the best reliable and available tool for spigelian hernia diagnosis.

2- Transverses abdomen is plane block (TPA) is the best regional methods of anesthesia used in treatment of spigelian hernia.

3- With the polene hernia system meshshort operative times short hospital stay no serious postoperative complication and no recurrence so this type of repair "tension free repair" is accepted for spigelian hernia.

\section{References}

Andersen, DK, 1986: Maingot's Abdominal Operations, $8^{\text {th }}$ edition, Vol. $1 \& 2$, Copyright and License information Disclaimer.

Bailey and Love's, 2000: Short Practice of Surgery $23^{\text {rd }}$ edition, RCG, Russell, NS, Williams, CJK, Bulstrode (eds.) London: Arnold.

Campos, SM, Walden, T, 1997: Images in clinical medicine spigelian hernia. N. Engl. J. Med. 336:1149-52.

Carter, JE, Mizes, C, 1992: Laparoscopic diagnosis and repair of spigelian hernia: Report of a case and technique. Am. J. Obstet. Gynecol. 167:77-8.

Fischer, JE, 2012: Fischer's Mastery of Surge- 
ry. $6^{\text {th }}$ edition, Vol. 1 , ISBN10 1469897180

Mittal, T, Kumar, V, Khullar, R, Sharma, A, Soni, $V$, et al, 2008: Diagnosis and management of Spigelian hernia: A review of literature and our experience. J. Mini. Access Surg. 4, 4:95100.

Moreno-Egea, A, Flores, B, Girela, E, Martín, J, Aguayo, J, et al, 2002: Spigelian hernia: Bibliographical study and presentation of a series of 28 patients. Hernia 6, 4:167-70.

Read, RC, 1978: Spigelian hernia. In: Nyhus M, Condon, RE (eds.): Hernia, $2^{\text {nd }}$ edition, Zest. Philadelphia: JB, Lippincott, USA

Skandalakis, JE, Gray, SW, Akin, JT, 1974: The surgical anatomy of hernial rings. Surg.
Clin. North Am. 54:1227-46.

Skandalakis, PN, Zoras, O, Skandalakis, JE, Mirilas, P, 2006: Spigelian hernia: surgical anatomy, embryology, and technique of repair. Am. Surg. 72:42-8.

Spangen, L, 1984: Spigelian hernia. World J. Surg. 13:573-80.

Stephen, W, Gray, SW, Skandalakis, JE, 1990: Atlas of Surgical Anatomy for General Surgeons 1985. London: Williams \& Wilkins.

Zaki, W, Alawad, AA, 2017: Spigelian hernia: A rare case report. Ann. Clin. Case Rept. 2.

Zimmerman, LM, 1994: Ventral hernia due to normal banding of the abdominal muscles. Surg. Gynecaol. Obset. 78:536-40.

Fig. 7: Marking site of spigelian hernia by dot line and site of incision.

\section{Explanation of figures}

Fig. 8: Hernial sac come out through defect in external oblique a penurosis mushroom like

Fig. 9: Excision sac and closure of defect in fascia transversalis and peritoneum.

Fig. 10: Prolene hernia system mesh prepared for insertion

Fig. 11: Circular part of prolone hernia system inserted between fascia tranversalis and transverses abdominis muscle.

Fig. 12: Defect external oblique aponeurosis closed around connected part of mesh.

Fig. 13: Suturing long tudenal superfacial part of mesh to external oblique aponeurosis
